\section{P138 HIGH STI PREVALENCE AMONG HIV-EXPOSED WOMEN PLANNING FOR PREGNANCY IN RURAL, SOUTHWESTERN UGANDA}

${ }^{1}$ Pooja Chitneni*, ${ }^{2}$ Mwebesa Bosco Bwana, ${ }^{3}$ Moran Owembabazi, ${ }^{4}$ Kasey O'Neil, ${ }^{3}$ Deogratious Tukwasibwe, ${ }^{3}$ Alice Najjuma, ${ }^{3}$ Cathy Kyampire, ${ }^{3}$ Sylvia Natukynda, ${ }^{3}$ Adolf Byamukama, ${ }^{3}$ Yona Mbalibulha, ${ }^{2}$ Paul Kato Kalyebara, ${ }^{5}$ Angela Kaida, ${ }^{6}$ Lynn Matthews. 'Brigham and Women's Hospital and Massachusetts General Hospital, Infectious Diseases, Boston, USA; ${ }^{2}$ Mbarara University of Science and Technology, Department of Internal Medicine, Mbarara, Uganda; ${ }^{3}$ Mbarara University of Science and Technology, Mbarara, Uganda; ${ }^{4}$ Massachusetts General Hospital, Center for Global Health, Boston, USA; ${ }^{5}$ Simon Fraser University, Vancouver, Canada; ${ }^{6}$ University of Alabama at Birmingham, Division of Infectious Diseases, Birmingham, USA

\subsection{6/sextrans-2019-sti.306}

Background Many HIV-affected couples desire children. STIs increase risks of infertility, poor maternal and infant outcomes, and HIV acquisition. We introduced STI testing in southwestern Uganda to characterize STI epidemiology among HIVexposed women planning for pregnancy.

Methods The Healthy Families PrEP study is enrolling 150 HIV-uninfected women planning for pregnancy with a partner known or suspected to be living with HIV. At enrollment, women are offered comprehensive safer conception counseling, including TDF/FTC as PrEP. We integrated STI testing for Chlamydia trachomatis (CT), Neisseria gonorrhea (NG), Trichomonas vaginalis (TV) (via GeneXpert), and Syphilis (via immunochromatographic rapid testing confirmed by RPR). We calculated STI prevalence and compared differences among women with and without STI (Fisher's exact test).

Results Between June 2018 and January 2019, 63 women completed baseline STI testing. Median age was 28 (IQR 2431) years. Seventeen participants (27\%) had STIs, including CT-14\%, NG-3\%, TV-8\%, Syphilis-6\%, and 5\% with two STIs. Women with STI were less likely to report prior pregnancy $(13 / 17(76 \%)$ vs. $45 / 46(98 \%), p=0.02)$ and trended towards being more likely to report prior stillbirth $(4 / 17$ $(24 \%)$ vs. $3 / 46(7 \%), p=0.08)$. Women with STI were less likely to report having relationship power to negotiate condom use $(7 / 17(41 \%)$ vs. $27 / 46(59 \%), p=0.26)$ and were younger (median age 26 vs. 29). PrEP uptake was high in both groups (16/17 (94\%) vs. 42/46 (91\%), p=1).

Conclusion We describe a $27 \%$ curable STI prevalence among HIV-exposed women planning for pregnancy. Women with STI were less likely to have had a prior pregnancy and trended towards prior stillbirth, possibly due to undiagnosed STI. In an HIV-endemic setting with social pressures to conceive children, infertility may contribute to increased HIV and STI exposures and prevalence. These data highlight the importance of integrating STI testing into HIV prevention programs to maximize the health of women, children, and families.

Disclosure No significant relationships.

\section{P139 THE RACIAL IMPACT OF AIDS TRENDS AMONG WOMEN IN SAO PAULO, BRAZIL}

Carmen Silvia Domingues*, Ângela Tayra, Marcia Polon, Mariza Tancredi. STI/AIDS Reference Center - Sao Paulo State Program of STI/AIDS, Sao Paulo Department of Health, Sao Paulo, Brazil

10.1136/sextrans-2019-sti.307

Background In the Sao Paulo State (SPS), the AIDS epidemic decreased among women, due to early diagnosis, antiretroviral use and prevention. This study aimed to analyze the trends of women living with AIDS (WLWA) and the HIV infection in pregnant women (PW-HIV) in the Sao Paulo City (SPC) and SPS, according to race/skin-color, from 2007-2016.

Methods Trend study using polynomial regression models performed with reported cases of WLWA and PW-HIV compared to SPC and SPS according to race/skin-color and diagnosis period. The annual number of WLWA and PW-HIV in each of the categories studied was considered as the dependent variable $(\mathrm{Y})$, and the independent variable $(\mathrm{X})$ was the time, represented by the calendar years, referring to the study period. The goodness of fit via $\mathrm{r}^{2}$ and $\mathrm{p}<0.05$ were used to determine which models and data were most appropriate.

Results In the period from 2007-2016, 18,571 AIDS cases in the SPS and 7,078 in the SPC reported among women were analyzed. In the SPS, WLWA presented a decreasing linear trend with first order modeling, with a higher fall rate among white women $\left[\mathrm{Y}=1,093-101 \mathrm{X} ; \mathrm{r}^{2}=0.97 ; \mathrm{p}<0.001\right]$ than in black $\left[\mathrm{Y}=237-15 \mathrm{X} ; \mathrm{r}^{2}=0.88 ; \mathrm{p}<0.001\right]$, and mixed color $/$ race $\left[\mathrm{Y}=600-35 \mathrm{X} ; \mathrm{r}^{2}=0.89 ; \mathrm{p}<0.001\right]$. In the SPC, the same trends were observed among white women $\left[\mathrm{Y}=362-34 \mathrm{X} ; \mathrm{r}^{2}=0.97\right.$; $\mathrm{p}<0.001]$, black $\left[\mathrm{Y}=108-6 \mathrm{X} ; \mathrm{r}^{2}=0.81 ; \mathrm{p}<0.001\right]$, and mixed color/race $\quad\left[Y=263-12 X ; r^{2}=0.86 ; p<0.001\right]$. For PW-HIV, 12,676 cases in the SPS and 4,160 in the SPC were analyzed. White PW-HIV showed a decreasing linear trend, being more expressive in the SPS $\left[\mathrm{Y}=681-21 \mathrm{X} ; \mathrm{r}^{2}=0,89 ; \mathrm{p}<0,001\right]$ than in the SPC $\left[Y=191-5 X ; r^{2}=0,64 ; p=0,005\right]$. In the SPS and the SPC, among black and mixed color/race PW-HIV the trend presented stability.

Conclusion To reduce the social inequalities, the care network must discuss specific strategies to improve the access to health care services and antenatal care services for black and mixed color/race WLWA and PW-HIV, prevention measures, sexual and reproductive health care, including access to long-acting contraceptives.

Disclosure No significant relationships.

\section{P140 THE RISING HIV EPIDEMIC AMONG KEY POPULATIONS: AN URGENT NEED FOR A FOCUSED TARGETED PREVENTION RESPONSE IN PAKISTAN}

${ }^{1}$ Faran Emmanuel, ${ }^{2}$ Baseer Achakzai, ${ }^{3}$ Tahira Reza. 'University of Manitoba, Centre for Global Public Health, Department of Community Health Sciences, WINNIPEG, Canada; ${ }^{2}$ National AIDS Control Program, Islamabad, Pakistan; ${ }^{3}$ Centre for Global Public Health, Islamabad, Pakistan

\subsection{6/sextrans-2019-sti.308}

Background HIV Surveillance data has been used extensively to guide HIV prevention program in Pakistan since 2004. The $5^{\text {th }}$ round of surveillance was conducted in 23 cities to determine the progression of the HIV epidemic, profile risk behaviors and program coverage among key populations to inform HIV prevention programs in Pakistan

Methods A total number of 5,660 FSWs, 6,773 MSM, 5,191 Transgenders and 4,062 PWIDs participated in the study using diverse sampling techniques to draw representative samples. Behavioral data were collected using structured questionnaires while blood samples were tested using two rapid HIV tests following WHO protocol. Informed consent was obtained and all participants were linked with HIV programs. Moreover, all positive subjects were linked to HIV treatment care and support. International ethical guidelines were followed and ethical 
approval for the study was obtained from PMRC ethics review board in Pakistan.

Results Overall, weighted prevalence of HIV among PWID was 38.4\% (95\% CI; 37.9, 38.9), 7.1\% (95\% CI; 6.8, 7.4) among TGs, 5.4\% (95\% CI: 5.2, 5.6) among MSM and 2.2\% (95\% CI: 2.1, 2.3) among FSWs. Among key HIV risk behaviors condom use at last sex was $27.7 \%$ in TGs, $50 \%$ in FSWs, 24\% in MSM and $15.8 \%$ in PWID, who also shared syringes extensively (31\%). Approximately 5\% of TG, 16.9\% of FSW and $4.0 \%$ of MSM reported having sex with a PWID in past year. Only 24.6\% PWID, 15\% TGs, 8.3\% FSWs and $13.3 \%$ MSM utilized HIV prevention programs in the past year, which explains the escalating trends of HIV among these populations.

Conclusion The HIV epidemic in Pakistan is growing rapidly and is following a rapidly propagating Asian HIV epidemic pattern. Pakistan needs to quickly scale-up services for key populations to contain the epidemic and to prevent a further spread to other KP members and into general population.

Disclosure No significant relationships.

\section{P141 FACTORS ASSOCIATED WITH HIV-RELATED STIGMA AMONG INDIVIDUALS ACCESSING ANTIRETROVIRAL THERAPY IN BRITISH COLUMBIA, CANADA}

${ }^{1}$ Andrea Bever*, 'Sean Grieve, 'Lu Wang, 'William Chau, 'Taylor Mclinden, ${ }^{1}$ Tim Wesseling, ${ }^{1}$ Kate Salters, ${ }^{2}$ Brittany Bingham, 'David Moore, ${ }^{1}$ Rolando Barrios. ${ }^{1} B C$ Centre for Excellence in HIVIAIDS, Vancouver, Canada; ${ }^{2}$ Vancouver Coastal Health, Aboriginal Health, Vancouver, Canada

\subsection{6/sextrans-2019-sti.309}

Background Despite public health messaging that antiretroviral therapy (ART) has improved health outcomes for people living with HIV (PLWH) and is effective in preventing HIV transmission, many PLWH continue to experience HIV-related stigma. It is critical to assess HIV-related stigma experienced by PLWH accessing ART in the modern HIV treatment era.

Methods The STOP HIV/AIDS Program Evaluation (SHAPE) study is a longitudinal cohort of PLWH $\geq 19$ years of age in British Columbia, Canada. This cross-sectional analysis uses SHAPE baseline survey data (collected January 2016-August 2018) and linked clinical registry data to examine factors associated with HIV-related stigma among individuals accessing ART. HIV-related stigma was self-reported using the ten-item Berger HIV stigma scale. Multivariable linear regression quantified the relationship between key explanatory variables and stigma.

Results Among 627 participants, 136(22\%) identified as women, $326(52 \%)$ were aged $\geq 50$ at enrolment, 374(60\%) identified as men who have sex with men, and 133(21\%) selfreported Indigenous ethnicity. The median stigma score was 47.5 (Q1-Q3: 32.5-62.5; range: 0-100). In the multivariable model, reporting injection drug use (IDU) in the past year $(\beta=4.54,95 \% \mathrm{CI}=0.23,8.86)$ or selecting "prefer not to answer" when asked about IDU history $(\beta=9.52,95 \% \mathrm{CI}=$ $4.77,14.28)$; experiences of lifetime violence $(\beta=7.62,95 \%$ $\mathrm{CI}=3.67,11.56)$; and having a mental health disorder diagnosis $(\beta=5.30,95 \% C I=1.88,8.73)$ were associated with higher stigma scores. Higher stigma scores were also associated with being 40-49 years old ( $\beta=6.21,95 \% \mathrm{CI}=1.58,10.85)$ compared to $<40$; age $\geq 50$ had no significant association. Living in a city with a population $\geq 100,000 \quad(\beta=-4.66,95 \% \mathrm{CI}=-$ $8.53,-0.78)$ was associated with lower stigma scores.

Conclusion Age, city size, IDU experience, violence, and mental illness were independently associated with HIV-related stigma. These findings provide support for an intersectional investigation into how these factors propagate stigma and how this experience impacts the health and wellbeing of PLWH in this setting.

Disclosure No significant relationships.

\section{P142 CHARACTERISTICS OF HIV-1 PRETREATMENT DRUG RESISTANCE AND ITS IMPACT ON COMBINED ANTIRETROVIRAL THERAPY IN BEIJING}

${ }^{1}$ Ruolei Xin*, ${ }^{1}$ Chun Huang, ${ }^{1}$ Lishi Bai, ${ }^{1}$ Hongyan Lu, ${ }^{2}$ Lijun Sun, ${ }^{2}$ An Liu. ${ }^{1}$ Beijing Center for Disease Prevention and Control, Beijing, China; ${ }^{2}$ Beijing You'an Hospital, Beijing, China

\subsection{6/sextrans-2019-sti.310}

Background From 2013, the expanded strategies for the initial of combined antiretroviral therapy (cART) were administrated in Beijing to the infected individuals whatever their CD4 cell counts, hence, the increasing trend of HIV-1 epidemic has been alleviated. Pretreatment drug resistance was monitored, yet its impact on cART and the derivation of acquired drug resistance should be well delineated.

Methods Treatment-naïve individuals with HIV-1 were recruited from September 2012 to April 2013 in Beijing You'an Hospital. The patients were followed up after the initial of cART. HIV-1 pol gene fragment was amplified using One-Step RT-PCR at stage of pretreatment and follow-up virological failure. The genotypic drug resistance was interpreted with Stanford University HIV Drug Resistance Database, with CPR algorithm and HIVdb program. The genotypes were determined using MEGA6.0 after multiple alignment by gene cutter, with reference to BLAST and RIP.

Results Totally, 324 treatment-naïve infections were recruited, and about 272 cases (84.0\%) were infected via MSM. The top three subtypes or CRFs were CRF01_AE (60.5\%), CRF07_BC (18.5\%) and B (15.4\%). 13 individuals were observed to possess PDR (4.0\%), at a low prevalence. The accumulative rate of virological failure was 9.5\%, which were observed in 31 cases, and the cohort maintenance rate of $75.4 \%$. The PDR induced virlological failure to develop acquired drug resistance (ADR) in three individuals, at a contributive rate of $23.1 \%$. Some persons gained virological failure at $0.3-0.6$ years post cART. Lower CD4 cell counts were prone to accompanied with the majority of virolgical failure (23.3\%). The dynamic fluctuation of acquired drug resistance were observed by single genome amplification.

Conclusion There was a low prevalence of transmitted drug resistance in treatment-naïve individuals in Beijing, and the TDR might convey $23 \%$ virological failure. The first-line cART obtained good performance of virological suppression, and the virological failure would be rectified by shift to second-line cART.

Disclosure No significant relationships. 